\title{
Attenuation of the suppressive activity of cellular splicing factor SRSF3 by Kaposi sarcoma-associated herpesvirus ORF57 protein is required for RNA splicing
}

\author{
VLADIMIR MAJERCIAK, MATHEW LU, XIAOFAN LI, and ZHI-MING ZHENG \\ Tumor Virus RNA Biology Section, Gene Regulation and Chromosome Biology Laboratory, Center for Cancer Research, National Cancer Institute, \\ National Institutes of Health, Frederick, Maryland 21702, USA
}

\begin{abstract}
Kaposi sarcoma-associated herpesvirus (KSHV) ORF57 is a multifunctional post-transcriptional regulator essential for viral gene expression during KSHV lytic infection. ORF57 requires interactions with various cellular proteins for its function. Here, we identified serine/arginine-rich splicing factor 3 (SRSF3, formerly known as SRp20) as a cellular cofactor involved in ORF57-

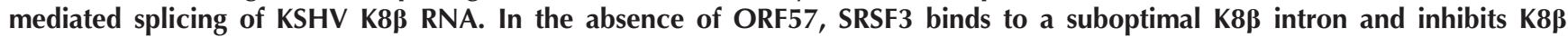
splicing. Knockdown of SRSF3 promotes K8 $\beta$ splicing, mimicking the effect of ORF57. The N-terminal half of ORF57 binds to the RNA recognition motif of SRSF3, which prevents SRSF3 from associating with the K8 $\beta$ intron RNA and therefore attenuates the suppressive effect of SRSF3 on K8ß splicing. ORF57 also promotes splicing of heterologous non-KSHV transcripts that are negatively regulated by SRSF3, indicating that the effect of ORF57 on SRSF3 activity is independent of RNA target. SPEN proteins, previously identified as ORF57-interacting partners, suppress ORF57 splicing activity by displacing ORF57 from SRSF3-RNA complexes. In summary, we have identified modulation of SRSF3 activity as the molecular mechanism by which ORF57 promotes RNA splicing.
\end{abstract}

Keywords: SRSF3; alternative splicing; RNA processing; ORF57; KSHV

\section{INTRODUCTION}

Kaposi sarcoma-associated herpesvirus (KSHV) encodes two potent proteins, ORF50 (or RTA) and ORF57 (or MTA), that enable robust expression of virus genes during lytic infection. ORF50 transactivates viral promoters for gene expression at the transcriptional level (Guito and Lukac 2012), whereas ORF57 assures that, after transcription, viral transcripts are correctly processed, stable, and translatable for protein production (Gupta et al. 2000; Kirshner et al. 2000; Majerciak et al. 2006; Majerciak and Zheng 2009). The importance of ORF57 in the KSHV life cycle was demonstrated by deletion of ORF57 from the virus genome, which led to the defective expression of viral lytic genes and abortive virus replication (Han and Swaminathan 2006; Majerciak et al. 2007). ORF57 promotes the post-transcriptional processing of a subset of viral transcripts and increases RNA stability, splicing, and translation of target transcripts (Boyne et al. 2010; Kang et al. 2011; Majerciak et al. 2011; Massimelli et al. 2011). Because ORF57 does not function as a bona fide export factor (Pilkington et al. 2012), it is unlikely to be directly involved

\footnotetext{
Corresponding author: zhengt@exchange.nih.gov

Article published online ahead of print. Article and publication date are at http://www.rnajournal.org/cgi/doi/10.1261/rna.045500.114. Freely available online through the RNA Open Access option.
}

in RNA export (Nekorchuk et al. 2007) as proposed (Boyne et al. 2008). Mechanistically, ORF57 forms ribonucleoprotein complexes with target RNAs and cellular cofactor(s) to provide its target specificity and to access various cellular pathways. We and others have identified multiple cellular proteins participating in ORF57 function, including SRSF1 (ASF/SF2), U2AF65, SM proteins, RBM15, OTT3, PABPC1, Aly/REF, CK II, hnRNP K, NXF1, CBP80, PYM, and others (Malik and Clements 2004; Malik et al. 2004; Majerciak et al. 2006, 2008, 2011; Boyne et al. 2010; Massimelli et al. 2011).

We previously showed that ORF57 is required for the splicing of several intron-containing KSHV transcripts (Majerciak et al. 2008). In KSHV-infected cells, ORF57 is enriched in the nuclear speckles and interacts with spliceosomal components. In the ribonucleoprotein complexes, ORF57 associates preferentially with the unspliced pre-mRNAs and small nuclear RNAs (U1, U2, U4, U5, and U6) and is involved in splice site selection and/or spliceosome assembly (Majerciak et al. 2008).

This article, published in $R N A$, is available under a Creative Commons License (Attribution-NonCommercial 4.0 International), as described at http://creativecommons.org/licenses/by-nc/4.0/. 
Serine/arginine-rich splicing factor 3 (SRSF3) is a member of a highly conserved family of splicing factors, the SR (serine/arginine-rich)-proteins (Zahler et al. 1992; Manley and Krainer 2010). By binding to RNA cis-elements in a doseand cell type-specific manner, SRSF3 regulates splicing of numerous cellular transcripts including its own mRNA (Jumaa and Nielsen 1997; Galiana-Arnoux et al. 2003; Jia et al. 2009; Sen et al. 2009; Wong et al. 2012). Moreover, SRSF3 regulates a wide range of other cellular processes including RNA polyadenylation (Lou et al. 1998; Maciolek and McNally 2007), RNA export (Huang and Steitz 2001; Escudero-Paunetto et al. 2010), pri-miRNA processing (Auyeung et al. 2013), genome stability (Loomis et al. 2009), and insulin signaling (Saeki et al. 2005). In mice, SRSF3 expression is essential for early embryogenesis, liver differentiation, and metabolic function (Jumaa et al. 1999; Sen et al. 2013). SRSF3 was recently identified as a protooncogene important for cell viability and growth (Jia et al. 2010), and increased expression of SRSF3 is common in many types of human cancers (Jia et al. 2010; He et al. 2011; Corbo et al. 2012; Iborra et al. 2013). SRSF3 overexpression leads to cell transformation, tumor induction, and the maintenance of tumor growth (Jia et al. 2010). Some reports also suggest a possible role for SRSF3 in the etiology of Alzheimer and bipolar diseases (Watanuki et al. 2008; Wong et al. 2012). In this report, we identify SRSF3 as a new ORF57 cofactor and demonstrate its suppression of RNA splicing.

\section{RESULTS}

\section{RBM15 and OTT3 inhibit ORF57- mediated RNA splicing of KSHV K8ß}

To investigate the role of cellular proteins in ORF57-mediated RNA splicing, we utilized a KSHV K8 $\beta$ cDNA (Tang and Zheng 2002; Yamanegi et al. 2005; Majerciak et al. 2008) in our in vivo splicing assay. The K $8 \beta$ cDNA was derived from a splicing intermediate of the $\mathrm{K} 8$ premRNA and retains a short K8 intron 2 with suboptimal $5^{\prime}$ and $3^{\prime}$ splice sites (Fig. 1A; Supplemental Fig. S1; Yamanegi et al. 2005). In the absence of ORF57, the K8 $\beta$ cDNA expresses K8 $\beta$ RNA, which encodes a K8 $\beta$ protein in size of 190 ami-

\section{$\mathbf{F}$} Hoechst dye. no acid (aa) residues by using a premature termination codon within the retained intron (Fig. 1A). Despite the presence of this premature termination codon, K8 $\beta$ mRNA survives nonsense-mediated decay (Yamanegi et al. 2005). In the presence of ORF57, splicing of the K $8 \beta$ intron leads to production of a fully spliced K8a RNA that encodes the 237 aa K8a (or KbZIP) protein (Fig. 1A; Majerciak et al. 2008). The difference
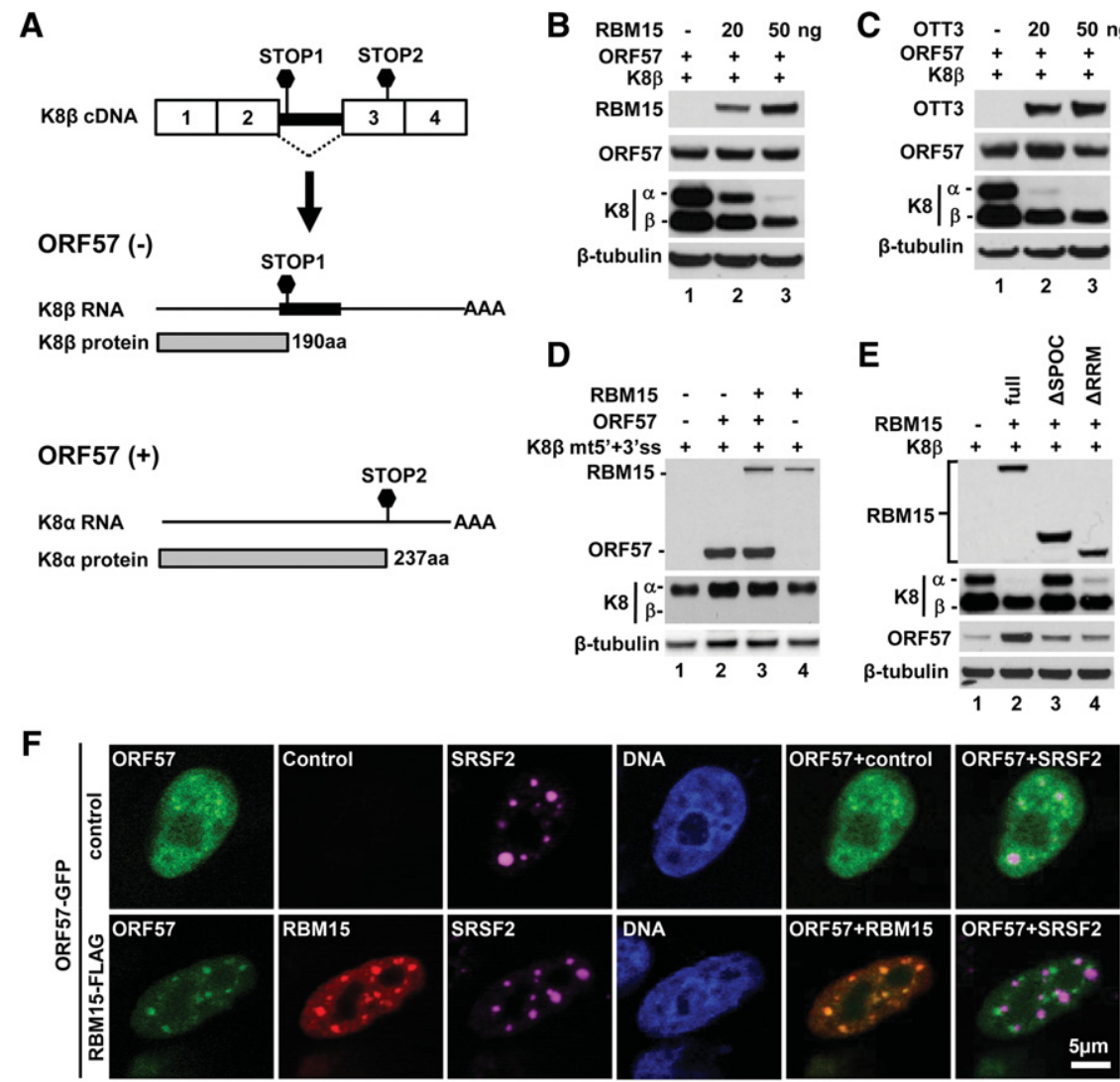

FIGURE 1. RBM15 protein suppresses ORF57-mediated RNA splicing of K8 $\beta$. (A) Diagram of $\mathrm{K} 8 \beta$ cDNA consisting of K8 exons 1-4 (numbered open boxes) and intron 2 (thick line). STOP1 and STOP2 represent two translational stop codons. Below the K8 $\beta$ cDNA diagram are two RNA species (K8 $\beta$ and $\mathrm{K} 8 \alpha$, thin lines) with stop codons produced either in the absence (-) or presence $(+)$ of ORF57 as indicated. K8 $\beta$ RNA produced in the absence of ORF57 encodes the K8 $\beta$ protein of 190 aa residues by using a stop codon (STOP1) from its retained intron, whereas K8a RNA lacking intron 2 is produced in the presence of ORF57 and encodes the K8a protein of 237 aa residues by using a stop codon (STOP2) in exon 3. $(B, C)$ Suppression of ORF57-mediated RNA splicing of K8 $\beta$ by RBM15 and OTT3. HEK293 cells were cotransfected at a fixed ratio (3:1) of K8 $\beta$ cDNA (pST3) and ORF57 (Majerciak et al. 2008) plus an increasing amount of RBM15 $(B)$ or OTT3 $(C)$ expression vectors as indicated above each panel. The levels of K8 $\beta$ splicing and coexpressed proteins at $24 \mathrm{~h}$ after transfection were determined by Western blotting with the corresponding antibodies. $(D)$ RBM15 does not suppress RNA splicing of the K8 $\beta$ intron with optimized $5^{\prime}$ and $3^{\prime}$ splice sites. Western blot analysis of K8 $\beta$ splicing from HEK293 cells transfected with the mutant $\mathrm{K} 8 \beta$ ( $\mathrm{pKY} 3, \mathrm{mt} 5^{\prime}+3^{\prime}$ ss) with or without cotransfection of ORF57 and RBM15. (E) The role of the RBM15 SPOC domain in inhibition of ORF57-mediated RNA splicing of K8 $\beta$. HEK293 cells were cotransfected with K8 $\beta$ and ORF57 expression vectors together with RBM15 lacking three $\mathrm{N}$-terminal RRMs $(\triangle \mathrm{RRM})$ or the $\mathrm{C}$-terminal SPOC domain ( $\triangle$ SPOC). Full-length wt RBM15 was used as a positive control and an empty vector was used as a negative control. K8 $\beta$ RNA splicing was determined by Western blotting using an anti-K8 antibody. (F) RBM15 displaces ORF57 from nuclear speckles. HeLa cells cotransfected with ORF57-GFP together with an empty (control) or RBM15-Flag expression vector were stained with anti-Flag and anti-SRSF2 (SC35) antibodies. Cellular nuclei were counterstained with 
in protein size between $\mathrm{K} 8 \alpha$ and $\mathrm{K} 8 \beta$ makes it possible to detect $\mathrm{K} 8 \beta$ splicing efficiency by Western blotting with an anti-K8 antibody that recognizes both $\mathrm{K} 8 \alpha$ and $\mathrm{K} 8 \beta$.

RBM15 and OTT3, two members of the SPEN ("splitend") protein family that form a complex with the cellular spliceosome (Zhou et al. 2002; Hiriart et al. 2005; Lindtner et al. 2006), are involved in the post-transcriptional processing (Uranishi et al. 2009). Both RBM15 and OTT3 interact with ORF57 and affect ORF57-mediated expression of the intronless KSHV ORF59 RNA (Majerciak et al. 2011). To determine whether RBM15 or OTT3 affects ORF57-mediated splicing, we cotransfected HEK293 cells with K8 $\beta$ and ORF57 expression vectors along with increasing amounts of RBM15 or OTT3 vector DNA. As shown in Figure 1, B and C, ORF57 enhanced K8 $\beta$ splicing in the absence of exogenous RBM 15 or OTT3, resulting in comparable levels of K8 $\alpha$ and $\mathrm{K} 8 \beta$ proteins (lane 1 in each panel). Unexpectedly, coexpression of RBM15 or OTT3 led to a substantial reduction of K8 $\beta$ splicing (Supplemental Fig. S2) and production of K8a protein in a dose-dependent manner, although it produced only a moderate effect on K8 $\beta$ expression (lanes 2 and 3 in panels Fig. 1B, C). This inhibition was observed with as little as $20 \mathrm{ng}$ of RBM15 plasmid DNA, which led to the expression of exogenous RBM15 at the endogenous level (Majerciak et al. 2011). This amount of exogenous RBM15 did not suppress ORF57 or K8 $\beta$ expression in separate cotransfection experiments (data not shown). Optimizing the K8 $\beta$ intron $5^{\prime}$ and $3^{\prime}$ splice sites (Yamanegi et al. 2005) enabled the optimized K8 $\beta$ to be spliced efficiently in the absence of ORF57, and RBM15 no longer suppressed the splicing or the production of $\mathrm{K} 8 \mathrm{a}$ (Fig. 1D). Therefore, we concluded that exogenous RBM15 and OTT3 inhibit ORF57-mediated splicing of K8 $\beta$ RNA bearing a suboptimal K8 $\beta$ intron.

All SPEN proteins consist of an N-terminal domain with three RNA-recognition motifs (RRMs) for RNA binding and a globular C-terminal SPOC domain for protein-protein interactions (Uranishi et al. 2009). To define the role of individual domains of RBM15 in the inhibition of ORF57-mediated splicing, RBM15 $\triangle$ RRM (deletion of aa 1-530), which lacks all three RRMs, and RBM15 $\triangle$ SPOC (deletion of aa 530-977) were examined in a K8 $\beta$ splicing assay in HEK293 cells. Both the full-length RBM15 and RBM15 $\triangle$ RRM efficiently inhibited K8 $\beta$ splicing (Fig. 1E, cf. lane 1 and lane 2 or 4), but RBM15 $\triangle$ SPOC did not (cf. lanes 1-3). This indicates that the RBM15 SPOC domain is essential and sufficient for suppression of ORF57-mediated K8 $\beta$ splicing, which is consistent with our previous report that the SPOC domain of RBM15 is an ORF57-interacting domain (Majerciak et al. 2011). Based on this observation, we speculated that binding of RBM15 to ORF57 interferes with the ability of ORF57 to interact with other cellular cofactors required for its splicing activity. To test this hypothesis, we used immunofluorescent staining to examine ORF57 localization in HeLa cells without or with ectopic RBM15 expression to see whether ectopic RBM15 would affect ORF57 colocalization with SRSF2
(SC35) speckles (Majerciak et al. 2008). HeLa cells were transfected with ORF57-GFP together with RBM15-Flag or empty vector (negative control). ORF57 partially colocalized with SRSF2 in the cells without RBM15-Flag (Fig. 1F, top panels), similar to what was previously observed in KSHV-infected cells (Majerciak et al. 2008). In contrast, ORF57 in the cells expressing RMB15-Flag failed to colocalize with the SRSF2 speckles, but instead colocalized with RBM15 granules. The examination of ORF57 colocalization with exogenous OTT3 in HeLa cells gave similar results to those obtained with RBM15 (data not shown). Together, these data provide the evidence that overexpressed SPEN proteins dislocate ORF57 from nuclear speckles and prevent ORF57 from interacting with cellular splicing factors.

\section{RBM15 disrupts ORF57 association with SRSF3}

To identify ORF57-binding partner(s) affected by RBM15, we performed anti-Flag-ORF57 immunoprecipitation (IP) and nano-liquid chromatography-tandem mass spectrometry (LC-MS/MS). Proteins pulled down with ORF57 from HEK293 cells without or with coexpression of RBM15-HA were stained with silver (Fig. 2A, left panel). Although both samples showed a comparable amount of ORF57 in the IPpulldowns, we identified a protein band of $\sim 20 \mathrm{kDa}$ that was present in the control sample without RBM15-HA, but was not present in the sample expressing RBM15-HA (Fig. $2 \mathrm{~A}$, arrows to the protein band by silver staining on the left panel and to the protein peak by ImageJ on the right panel), indicating loss of its interaction with ORF57 in the presence of RBM15-HA. Proteomic analysis of the corresponding protein band regions from both samples (Supplemental Table S1) identified it as SRSF3, the smallest member of the SR protein family. We subsequently confirmed by IP followed by Western blot analyses that ORF57 indeed interacts with endogenous SRSF3 (Fig. 2B, lane 5) and this interaction in HEK293 cells is prevented by ectopic RBM15 (Fig. 2B, cf. lanes 5 and 6). The ORF57-SRSF3 interaction is not an RNA-mediated interaction because it occurred in cell extracts digested by RNase A/T1 prior to the pulldown. By mixing proteins individually expressed from HEK293 cells for coIP and Western blot analyses, we demonstrated that, although SRSF3-Flag and RBM15-HA do not interact with each other (Fig. 2C, lanes 2, 4, 6, and 8) in the presence or absence of ORF57, their binding to ORF57 is mutually exclusive (Fig. 2C, cf. lane 9 to lanes 10 and 11). Interestingly, we found that endogenous RBM15 in B cells interacts with ORF57 (data not shown), its levels were reduced as expression of SRSF3 increased over the time of viral lytic infection and the expression of ORF57 (Supplemental Fig. S3A).

To confirm the negative effect of ectopic RBM15 on ORF57-SRSF3 interaction in vivo, we coexpressed ORF57GFP in HeLa cells with SRSF3-Flag in the absence or presence of RBM15-HA and visualized their colocalization with immunofluorescent staining (Fig. 2D). In the control cells without 
A

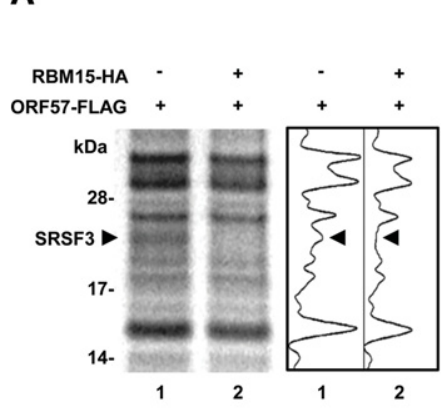

B

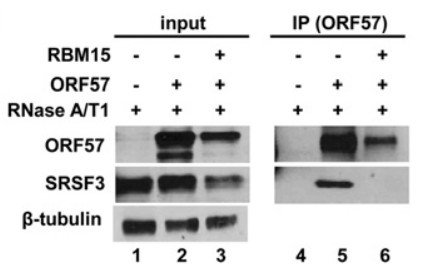

D

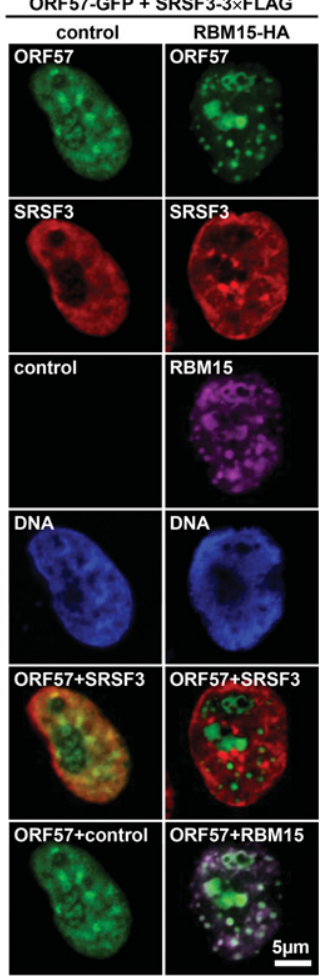

C

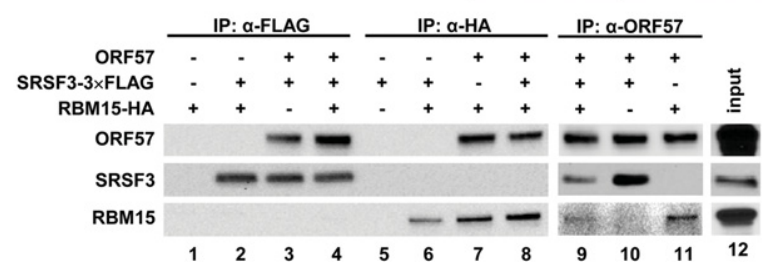

FIGURE 2. RMB15 disrupts interaction of ORF57 with cellular splicing factor SRSF3. (A) HEK293 cells were cotransfected with ORF57-Flag together with an empty or RBM15-HA expression vector. ORF57-associated proteins were pulled down with an anti-Flag antibody, separated with SDS-PAGE, visualized with silver staining, and analyzed by ImageJ software. (B) Western blot analysis of endogenous SRSF3 coimmunoprecipitated with ORF57 from HEK293 cells with or without coexpression of RBM15-HA protein. All cell lysates were treated first with RNase A/T1 before the coimmunoprecipitation (IP) with a polyclonal rabbit anti-ORF57 antibody and detected by Western blotting with anti-Flag for ORF57 and 7B4 for SRSF3. $(C)$ In vitro interactions among ORF57, SRSF3, and RBM15. Flag-tagged SRSF3, HA-tagged RBM15, and untagged ORF57 were individually expressed in HEK293 cells. Individual cell extracts treated with RNase A/T1 were mixed and incubated on ice for $30 \mathrm{~min}$. An extract from untransfected HEK293 cells was used to adjust the reaction volume. The interacting proteins in a protein complex were coimmunoprecipitated with a mouse monoclonal anti-Flag M2 (for SRSF3), anti-HA (for RBM15), or anti-ORF57 antibody and detected by Western blotting with the corresponding rabbit polyclonal antibodies. $(D)$ The effect of ectopic expression of RBM15 on colocalization of ORF57 with SRSF3. HeLa cells were cotransfected with ORF57-GFP and SRSF3-3×Flag together with an empty vector (control) or RBM15-HA expression vector. Subcellular localization of SRSF3 and RBM15 was determined by immunofluorescence, and cellular nuclei were counterstained with Hoechst dye.

ectopic RBM15-HA, we observed a high degree of ORF57 colocalization with SRSF3 in the nucleoplasm and in the nuclear speckles, confirming the interaction we found with co-
IP. In contrast, ORF57 did not colocalize with SRSF3 in the cells coexpressing RBM15-HA, but rather appeared as distinct granular forms with RBM15 (Fig. 2D), as also seen in Figure 1F. SRSF3 in these cells remained mainly in the nucleoplasm outside of RBM15-ORF57 granules. Taking these results together, we concluded that the interaction of SRSF3 and ORF57 in cells can be interrupted by ectopic RBM15.

\section{The N-terminus of ORF57 binds to the RRM domain of SRSF3}

To identify the mutual interaction domains of ORF57 and SRSF3, we performed co-IP experiments with a set of ORF57 and SRSF3 mutants (Fig. 3). At 164 aa, SRSF3 is the smallest member of the human SR protein family and, like all other SR proteins, is composed of an N-terminal RNA-recognition motif (RRM, aa 10-83) and a C-terminal RS (arginine/serine-rich) domain (aa 86-164) connected by a short hinge region (Zahler et al. 1992; Jia and Zheng 2012). Using the two SRSF3 truncation mutants, we found that the Nterminal RRM domain of SRSF3 is required for interactions with ORF57, whereas its RS domain, which is responsible for the protein-protein interaction of SR proteins (Caceres and Krainer 1993), is not (Fig. 3A, cf. lanes 7 and 8).

The N-terminal half of ORF57 is unstructured and is distinct from its highly structured C-terminal half (Majerciak and Zheng 2009). The N-terminal half of ORF57 harbors three redundant nuclear localization signals (NLSs) that are

A
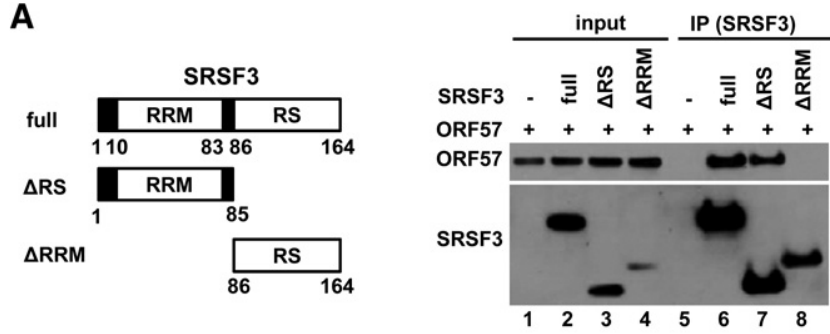

B
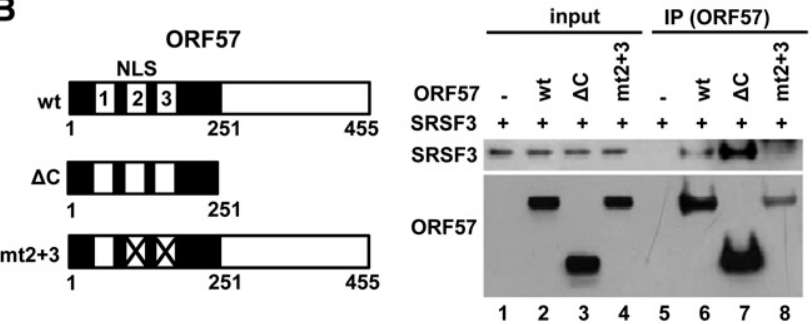

FIGURE 3. Mapping of ORF57 and SRSF3 interaction domains. Diagrams on the left show the structures of Flag-SRFS3 $(A)$ and ORF57-Flag $(B)$ and their mutants used in IP pulldown assays. Numbers below the diagrams represent the amino acid residues of the RNA-recognition motif (RRM), arginine/serine-rich domain (RS), and nuclear localization signal (NLS) in the full-length protein. On the right of each panel are Western blots of untagged ORF57 pulled down with Flag-SRSF3 $(A)$ or SRSF3-T7 pulled down with ORF57Flag $(B)$ from HEK293 cell extracts using an anti-Flag antibody. Cell lysates treated with RNase A/T1 were used in each IP pulldown assay. 
essential for many ORF57 activities (Majerciak et al. 2006), including its increase of RNA splicing (Majerciak et al. 2008). To map the SRSF3 interaction domain in ORF57, we examined two ORF57 mutants, a C-terminal truncation mutant $(\Delta \mathrm{C}$, aa 1-251) and a functionally inactive full-length ORF57 with point mutations in NLS 2 and 3 (ORF57 $\mathrm{mt} 2$ +3 ), along with wild type (wt) ORF57 (Fig. 3B, diagram). We compared the activities of these two mutants for binding SRSF3. The $\triangle \mathrm{C}$ mutant, but not the $\mathrm{mt} 2+3$ mutant, retained high binding affinity for SRSF3 (Fig. 3B, cf. lanes 7 and 8), although both ORF57 mutants are nuclear proteins (Majerciak et al. 2006). These data indicate that the domain of ORF57 that interacts with SRSF3 resides in its N-terminal half, which contains the NLSs. This observation is consistent with our previous findings that the ORF57 $\mathrm{N}$-terminal half is responsible for the binding of other cellular cofactors (Majerciak et al. 2006, 2011; Massimelli et al. 2013).

\section{SRSF3 is a negative regulator for RNA splicing of K8ß}

Given the fact that RBM15 inhibits ORF57-mediated K8 $\beta$ splicing and interrupts the ORF57-SRSF3 interaction, we further investigated the possible roles of SRSF3 in the regulation of ORF57-mediated RNA splicing of K8 $\beta$. We used siRNA to knock down SRSF3 in HEK293 cells transfected with a K8 $\beta$ expression vector alone or together with an ORF57 expression vector (Fig. 4A), and found that knocking down SRSF3 expression in HEK293 cells in the absence of ORF57 led to a substantial (30-fold) increase of K8 $\beta$ splicing (to $20.2 \%$ ) over the minimal splicing level $(0.6 \%)$ in control cells treated with a nontargeting (NT) siRNA (Fig. 4A, cf. lanes 1 and 2). In contrast, SRSF1 (ASF/SF2) knockdown showed no visible effect (1.0\%), and knockdown of RBM15 produced only a minimal increase of K8 $\beta$ splicing (4.0\%, 6.7-fold) (Fig. 4A, lanes 3 and 4$)$. These data clearly indicate that SRSF3 suppresses K8 $\beta$ splicing. Apparently, ectopic ORF57 increases the expression of endogenous SRSF3 and K8 $\beta$ splicing (Fig. 4A, cf. lanes 5 and 1; Supplemental Fig. S3B), but knocking down SRSF3 expression in HEK293 cells in the presence of ORF57 (Fig. $4 \mathrm{~A}$, lanes 5-8), as expected, further increased (approximately threefold) K8 $\beta$ splicing mediated by ORF57 (Fig. 4A, cf. lanes 6 and 5), although ORF57 levels were also substantially reduced in the cells with SRSF3 knockdown (lane 6). In contrast, RBM15 knockdown had no effect on the K8 $\beta$ splicing or ORF57 expression (lanes 8), but SRSF1 knockdown reduced ORF57 expression and its splicing activity by approximately twofold (Fig. 4A, cf. lanes 7 and 5).

Next, we examined the effect of increasing SRSF3 level on K8 $\beta$ splicing using transient expression of SRSF3-T7 in HEK293 cells in the presence or absence of ORF57. Ectopic SRSF3-T7 increased the expression, but not the RNA splicing, of K8 $\beta$ in the absence of ORF57 (Fig. 4B, lanes 1 and 2). When expressed in the presence of ORF57, the ectopic SRSF3-T7 strongly suppressed ORF57-mediated splicing of K8 $\beta$ RNA (Fig. 4B, cf. lanes 4 and 3). These data further reinforce that
A

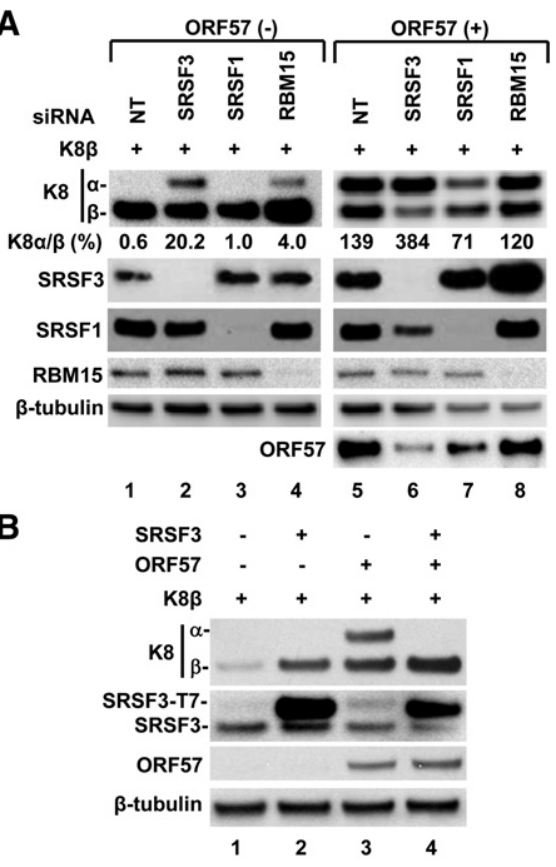

C

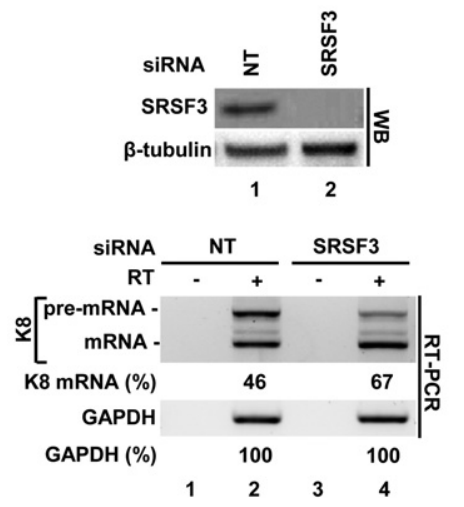

FIGURE 4. Negative regulation of K8 $\beta$ splicing by SRSF3. (A) The effect of knockdown of endogenous SRSF3, SRSF1, or RBM15 proteins on K8 $\beta$ splicing in the absence or presence of ORF57. HEK293 cells were pretreated with nontargeting (NT) or gene-specific siRNAs followed by cotransfection of a K8 $\beta$ expression vector along with an ORF57 vector or empty expression vector. Knockdown efficiency and K8 $\beta$ splicing for K8a expression were determined by Western blotting. Cellular $\beta$-tubulin was used as a loading control. (B) The negative effect of ectopic expression of SRSF3 on ORF57-mediated K8 $\beta$ splicing. HEK293 cells were transfected with a K8 $\beta$ expression vector, with or without cotransfection of ORF57 and SRSF3-T7 expression vectors. Ectopically expressed proteins and K8 $\beta$ RNA splicing for K8a expression were detected by Western blotting. Cellular $\beta$-tubulin served as a loading control. (C) SRSF3 knockdown promotes K8 $\beta$ splicing during KSHV infection. The efficiency of SRSF3 knockdown in Bac36 $\Delta 57$ cells carrying an ORF57-null KSHV genome was determined by Western blotting (WB). Cellular $\beta$-tubulin served as a loading control. RT-PCR analysis of K8 RNA splicing was performed on total RNA extracted from Bac $36 \Delta 57$ cells treated twice with an NT or SRSF3 siRNA followed by induction of the KSHV lytic cycle by treatment with sodium butyrate for 24 h. The samples without reverse transcriptase (RT-) were used to confirm specific RNA amplification. K8 RNA splicing efficiency (\%) was calculated as K8 mRNA divided by the sum of K8 mRNA and pre-mRNA. Cellular GAPDH RNA served as a loading control, and its expression in the presence of NT was set to $100 \%$. 
SRSF3 functions as a negative regulator of ORF57-mediated K8 $\beta$ splicing.

To test whether SRSF3 negatively regulates K8 $\beta$ splicing in the context of the viral genome during native KSHV infection, we knocked down SRSF3 in HEK293 cells stably infected with an ORF57-null KSHV genome (Bac36 $\Delta 57)$. The efficiency of SRFS3 knockdown by siRNA was determined by Western blot (WB) (Fig. 4C, top panel) and the level of K8 splicing was determined by RT-PCR (Fig. 4C, lower panel). The Bac $36 \Delta 57$ cell line was chosen in this study because of its deficiency of viral RNA splicing due to a lack of ORF57 during viral lytic reactivation (Majerciak et al. 2008). In the cells treated with a control NT siRNA, 46\% of K8 RNA was spliced (Fig. 4C, lane 2 in lower panel), whereas the K8 RNA splicing increased to $67 \%$ in the cells with siRNA knockdown of SRSF3 expression (Fig. 4C, cf. lanes 4 and 2 in the lower panel), leading to less K8 pre-mRNA. Together, the data from transient expression of HEK293 and KSHV infection of Bac $36 \Delta 57$ cells further indicate that SRSF3 functions as a negative regulator of K8 RNA splicing, and its negative activity on RNA splicing can be attenuated by ORF57.

\section{SRSF3 preferentially binds to $\mathrm{K} 8 \beta$ intron}

Given our finding that SRSF3 is an RNA-binding protein and interacts with ORF57 through its N-terminal RRM domain, we next investigated whether ORF57 binding to SRSF3 affects SRSF3 binding to K8 $\beta$ RNA. To do this, we performed RNA UV crosslinking and immunoprecipitation (RNA-CLIP) assays using HEK293 cells expressing K8 $\beta$ RNA in the presence or absence of ORF57 and SRSF3-Flag. Again, we found that ORF57-mediated K8 $\beta$ splicing to produce K8a RNA could be suppressed by exogenous SRSF3-Flag (Fig. 5A input, cf. lanes 2 and 3). After UV irradiation-induced RNA-protein crosslinking in the cells, SRSF3-associated K8 $\beta$ RNA was pulled down by an anti-Flag antibody and detected by RTPCR with a K8-specific primer pair (Fig. 5A) from cells with (lane 3) or without (lane 4) ORF57 expression, indicating ORF57-independent binding of SRSF3 to K8 $\beta$ RNA. This result is consistent with the observation that ORF57 binds to unspliced K8 $\beta$ RNA in the presence of cellular proteins, but not to fully spliced K8a RNA (Majerciak et al. 2008). The binding of both ORF57 and SRSF3 to K8 $\beta$ RNA suggests the importance of the intronic sequences in the regulation of K8 $\beta$ splicing by ORF57 and SRSF3. We initially examined this hypothesis by swapping the K $8 \beta$ intron into the coding region of GFP and detecting the splicing efficiency of the chimeric GFP RNA in HEK293 cells in the presence or absence of ORF57 expression (Fig. 5B). We found that the splicing efficiency of the GFP RNA was increased in the cells expressing ORF57 over the cells lacking ORF57 (Fig. 5B, cf. lanes 4 and 2 ), indicating that the intron sequence of $K 8 \beta$ is targeted by ORF57 to promote RNA splicing in a heterologous RNA.

To better understand the molecular interplay between ORF57 and SRSF3 in binding to the K8 $\beta$ intron, we per-

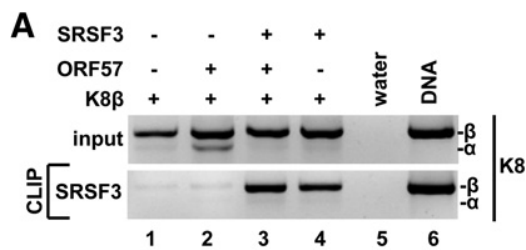

B
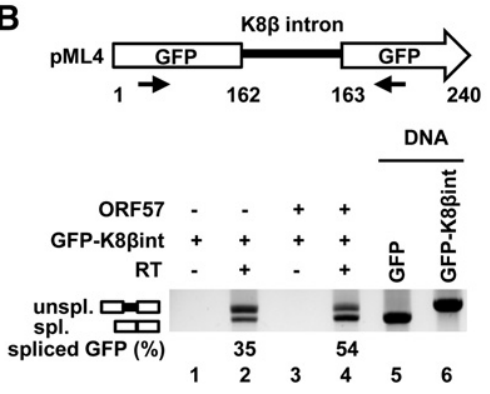

FIGURE 5. Association of SRSF3 with K $8 \beta$ RNA regulates splicing of K8 $\beta$ intron. (A) Association of SRSF3 with K8 $\beta$ RNA in vivo. HEK293 cells were cotransfected with K8 $\beta$, ORF57, and/or SRSF3-Flag expression vectors. K8 RNA pulled down with SRSF3 in RNA CLIP assays using an anti-Flag antibody was analyzed by RT-PCR using a sense primer from exon 1 (oST1) and an antisense primer from exon 3 (oST3) (Tang and Zheng 2002). (B) K8 $\beta$ intron in a heterologous RNA is susceptible to ORF57 regulation. HEK293 cells were cotransfected with pML4, a GFP expression vector containing the entire $\mathrm{K} 8 \beta$ intron inserted into the GFP open reading frame, along with an ORF57 expression vector or an empty vector. Total cell RNA extracted $24 \mathrm{~h}$ after transfection was monitored for GFP RNA splicing by RT-PCR with the indicated primers (black arrows). The RNA splicing efficiency (\%) was calculated as described for Figure 4C.

formed RNA pulldown and Western blot assays using a series of RNA oligos spanning the entire K8 $\beta$ intron and its flanking exon sequences (Fig. 6A diagram; Supplemental Table S2; Supplemental Fig. S1). Considering the suppressive effect of SRSF3 on K8 $\beta$ splicing in both the absence and the presence of ORF57, we first tested the binding of endogenous SRSF3 to the RNA oligos using whole-cell lysates of HEK293 cells with or without ORF57 expression. As shown in the lower panels of Figure 6A, SRSF3 bound to a region adjacent to the exon 2-intron $5^{\prime}$ splice site (oVM241, lane 2) and an intron region immediately upstream of the intron polypyrimidine tract (PPT) (oVM 243 and oVM244, lane 4 and lane 5 ), but did not bind to the sequences downstream from its $5^{\prime}$ splice site (oVM242, lane 3 ) and bound very weakly to the PPT of its $3^{\prime}$ splice site (oVM245, lane 6). The pattern was the same in cell lysates without and with ORF57. The RNA oligo sequence covering the intron $3^{\prime}$ splice site-exon 3 junction did not show any binding to SRSF3 (oVM246, lane 7). Similar binding profiles were also obtained with exogenously expressed SRSF3 in the absence and presence of ORF57 (data not shown). Interestingly, we found that ORF57 bound to all of the examined RNA regions, with a binding affinity of the corresponding RNA oligos in the order of oVM244>oVM241/243>oVM242>oVM245/oVM246 (Fig. 6A). Increased ORF57 binding to oVM244, which corresponds to an intron region that covers a putative branch point 
A

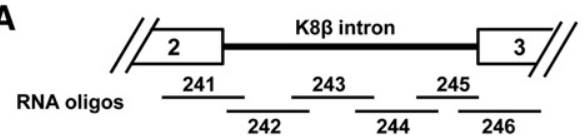

C
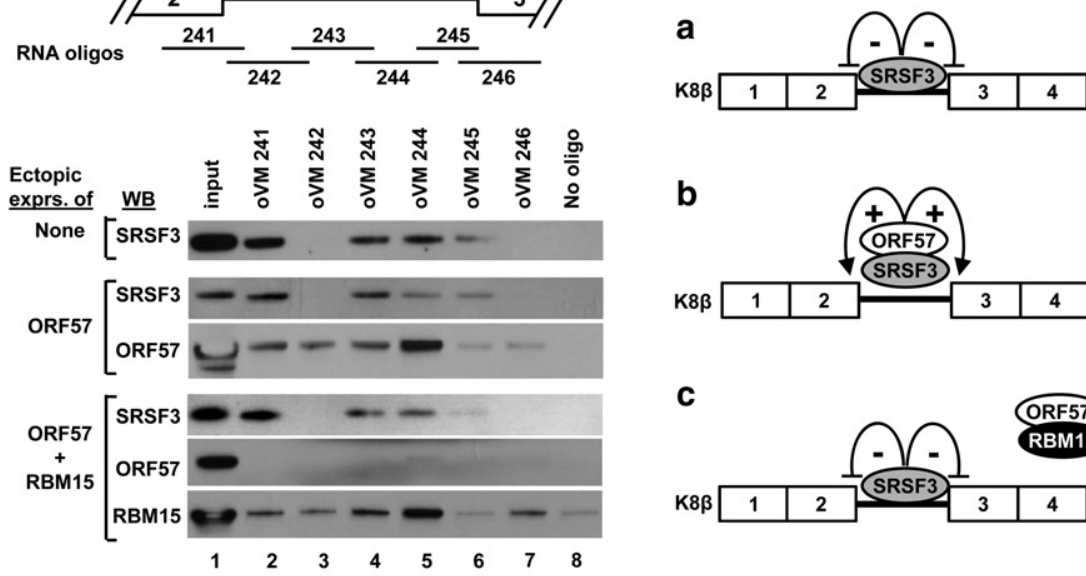

B
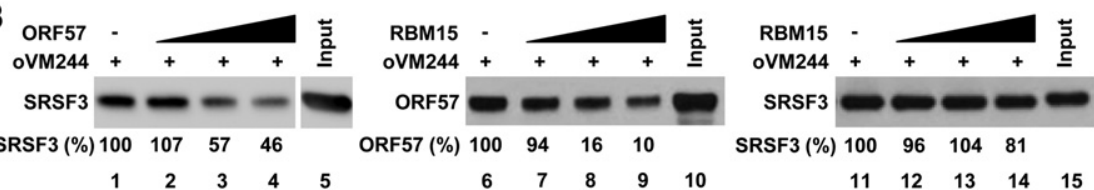

FIGURE 6. Binding of SRSF3, ORF57, and RBM 15 to K8 3 intron. (A) In vitro assays of SRSF3, ORF57, and RBM 15 binding to the intronic regions of K8 $\beta$ RNA. Shown on the top are a diagram of the K8 $\beta$ intron (thick black line) with the surrounding K8 exons (numbered open boxes) and biotin-labeled RNA oligos (numbered lines) spanning the entire K8 $\beta$ intron. Below the diagram are Western blot results of endogenous SRSF3 and ectopically expressed ORF57 and RBM15 proteins from HEK293 cell extracts obtained from the RNA pulldowns with individual RNA oligos. (B) Dose-dependent inhibition of SRSF3-RNA interaction by ORF57 and of the ORF57-RNA interaction by RBM15. In the left panel, cell extracts prepared from HEK293 cells expressing SRSF3-3×Flag were incubated with RNA oligo oVM244 for $2 \mathrm{~h}$, followed by the addition of increasing amounts $(0,10,100,1000 \mathrm{ng})$ of purified recombinant ORF57. Alternatively in the right two panels, cell extracts prepared from HEK293 cells expressing ORF57-Flag or SRSF3-3×Flag were mixed with equal volumes of extracts from cells transfected with various amounts $(0$, 250, 500, and $1000 \mathrm{ng}$ ) of RBM15-Flag vector DNA. After 30-min incubation at room temperature, RNA oligo oVM244 was added. Proteins bound to the RNA oligo after overnight incubation at $4^{\circ} \mathrm{C}$ were examined by Western blotting. $(C)$ Proposed model of how SRSF3 regulates K8 $\beta$ splicing in the absence of ORF57 (a), in the presence of ORF57 (b), and in the presence of ORF57 and ectopic RBM15 (c).

region immediately upstream of the $\mathrm{PPT}$, appeared to reduce SRSF3 binding to the same region (oVM244, lane 5). Further study demonstrated that ORF57 reduces the binding of SRSF3 to this region of the RNA (oVM244) in a dose-dependent manner (Fig. 6B, the left panel). Together, these data suggest that SRSF3 selectively interacts with an RNA region independently of ORF57, whereas the binding of ORF57 to the RNA may (oVM241, 243, and 245) or may not (oVM242 and 246) depend on SRSF3 or may even compete with SRSF3 for the binding (oVM244).

\section{Ectopic RBM15 prevents the association of ORF57, but not SRSF3, with the K8ß intron}

Given the suppressive effect of exogenous RBM15 on ORF57mediated K8 $\beta$ splicing and on the ORF57-SRSF3 interaction, we next investigated the effect of ectopically expressed RMB15 on the binding of SRSF3 and ORF57 to the K8 $\beta$ RNA oligos. Exogenous RBM15 bound almost every examined oligos (ex- cept oVM245), with variable binding affinity beyond the background (Fig. 6A). Binding to RNA oligo oVM245, which covers the PPT, was at background levels similar to the no oligo control (cf. lanes 6 and 8). Interestingly, RBM15 binding to RNA was found to prevent ORF57, but not SRSF3, from interacting with the RNA (cf. the ectopic ORF57 alone versus ectopic ORF57 and RBM15). This RBM15-specific effect on the binding of ORF57 to K8 $\beta$ intron RNA was dose-dependent, whereas the effect on SRSF3 was less so (Fig. 6B, two panels on the right).

Based on these findings and our previous results on the RBM15-ORF57 interaction (Majerciak et al. 2011), we propose a working model of how ORF57 may regulate K8 $\beta$ RNA splicing (Fig. $6 \mathrm{C})$. In this model, endogenous SRSF3 normally binds to the K8 $\beta$ intron to inhibit the intron splicing (a). Upon expression, ORF57 interacts with SRSF3 to prevent SRSF3-RNA interaction and attenuates the suppressive effect of SRSF3 on K8 $\beta$ intron splicing (b). Increased amounts of RBM15 disrupt the ORF57SRSF3 and ORF57-RNA interactions, leading to restoration of SRSF3's suppressive activity on K8 $\beta$ intron splicing (c).

\section{ORF57 promotes RNA splicing of BPV-1 and HPV16 late transcripts by attenuating SRSF3 activity}

To determine whether ORF57 regulates other SRSF3-dependent splicing events, we expressed the late transcripts of bovine papillomavirus type $1(\mathrm{BPV}-1)$ and human papillomavirus type 16 (HPV16), which are both regulated by SRSF3 (Jia et al. 2009). In both cases, SRSF3 binds to A/C-rich elements within viral late transcripts and inhibits RNA splicing (Jia et al. 2009). In BPV-1, SRSF3 binding to splicing enhancer 4 (SE4) inhibits the usage of the nucleotide (nt) $36053^{\prime}$ ss and promotes alternative RNA splicing at the nt 3225 3'ss (diagram in Fig. 7A). Similarly, SRSF3 binds to an exonic splicing enhancer (ESE) in HPV16 late transcripts and inhibits splicing of an intron immediately downstream (diagram in Fig. 7B). To assess ORF57's ability to regulate splicing of these SRSF3-dependent introns, HEK293 cells were cotransfected with a subgenome construct containing a BPV-1 or HPV16 late gene region with or without cotransfection of an ORF57 expression vector. RNA splicing in the transfected cells was determined by RT-PCR using primers spanning the intron (Pr1 $+\operatorname{Pr} 2$ ), and the total level of viral transcripts was determined 
A
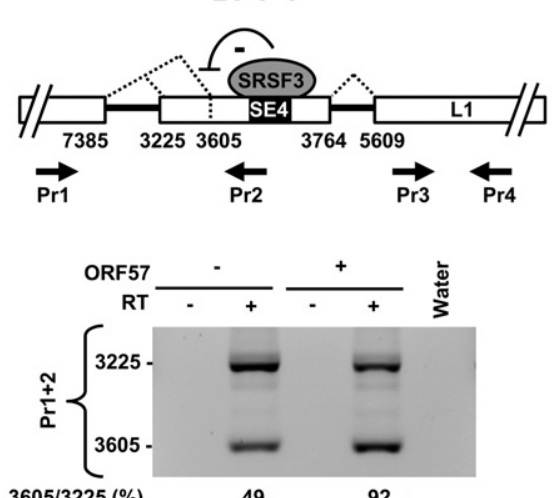

$3605 / 3225(\%) \quad 49 \quad 92$

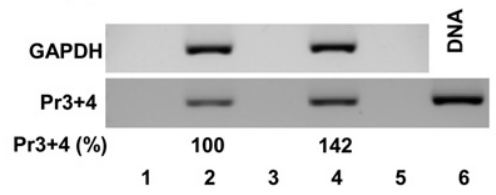

B
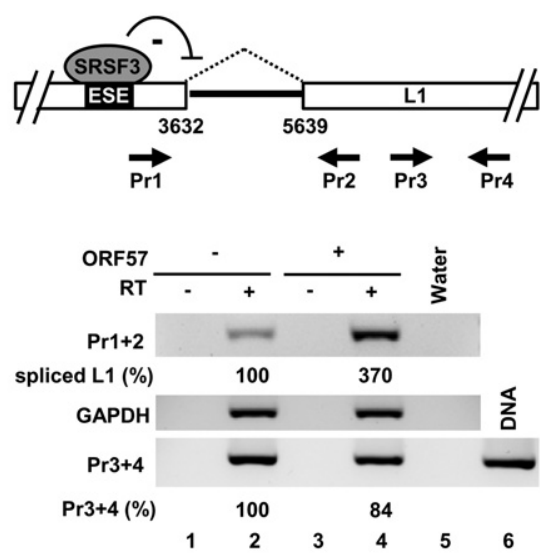

C

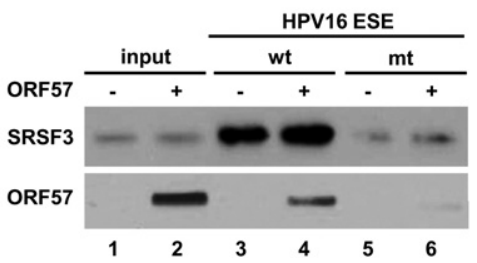

FIGURE 7. ORF57 promotes SRSF3-regulated RNA splicing of BPV-1 and HPV16. (A) ORF57 promotes RNA splicing of BPV-1 late transcripts. Diagram of the late gene (L2L1) region of a BPV-1 minigene (pXLF1) containing splicing enhancer 4 (SE4, black box), which binds SRSF3. Binding of SRSF3 to SE4 inhibits the usage of the nt 3605 3'ss, resulting in increased usage of an upstream nt $32253^{\prime}$ ss. Below the diagram are RT-PCR results of BPV-1 late transcripts from HEK293 cells with or without ORF57 expression. The usage of an alternative 3 'ss in the RNA splicing was monitored with a primer set $\operatorname{Pr} 1+\operatorname{Pr} 2$ and was calculated as a ratio (\%) of splicing at nucleotides $3605 / 3225$ based on the signal density of each amplicon. The total amount of BPV1 RNA was determined with Pr3 + Pr4 after normalization to GAPDH RNA level. (B) ORF57 promotes RNA splicing of HPV16 late transcripts. Diagram on the top shows the late region of an HPV16 minigene (pJR5) with an exonic splicing enhancer (ESE, black box) binding SRSF3. Below the diagram are RT-PCR results of HPV16 late RNA splicing in HEK293 cells with or without ORF57 expression. The level of spliced RNA and total amount of HPV16 late RNA were determined by RT-PCR with the primer sets of PR1 + Pr2 and Pr3 + Pr4, respectively. Changes in RNA splicing level (\%) as a result of ORF57 expression were calculated after normalization to the total amount of HPV16 RNA (Pr1 + Pr2/Pr3 + Pr4). Total HPV16 RNA was calculated after normalization to GAPDH RNA. (C) Binding of SRSF3 and ORF57 to HPV16 ESE. Western blot analysis of endogenous SRSF3 and ectopically expressed ORF57-Flag from HEK293 cell extracts obtained from the RNA pulldowns with a wt or mt ESE RNA oligo.

by primers from downstream exons $(\operatorname{Pr} 3+\operatorname{Pr} 4)$ (Supplemental Table S3). BPV-1 late transcripts in the cells were normally spliced more often at the nt $32253^{\prime}$ ss than that at the nt 3605 $3^{\prime}$ ss, with a ratio of $3605 / 3225$ to $\sim 49 \%$ (Fig. 7A, lane 2) as previously reported (Jia et al. 2009). However, ORF57 coexpression in the cells reduced the RNA splicing at the nt $32253^{\prime}$ ss and increased both the splicing at the nt $36053^{\prime}$ ss, with the $3605 / 3225$ splicing ratio to $\sim 92 \%$, and the production of total BPV-1 late transcripts $(\sim 142 \%$, Fig. 7 A, lane 4$)$. Similarly, HPV16 late transcripts showed a $\sim 370 \%$ increase of intron splicing in the cells with ORF57 coexpression over the cells without ORF57 (Fig. 7B, cf. lanes 2 and 4), while the overall HPV16 expression remained mainly unchanged in both conditions (100\% versus $84 \%$ ). By RNA pulldown assays, we further confirmed the binding of SRSF3 and ORF57 to HPV16 wt ESE, but only weakly to its mutant (Fig. 7C, lanes 3-6). These data clearly indicate that ORF57 promotes RNA splicing of SRSF3-regulated transcripts in general, without significantly altering overall RNA expression, consistent with what we had previously observed by knocking down SRSF3 expression (Jia et al. 2009).

\section{DISCUSSION}

In this report, we investigated how ORF57 promotes RNA splicing and identified SRSF3 as a host factor involved in ORF57-mediated splicing. We found that SRSF3 generally suppresses K8 $\beta$ RNA splicing via its binding to the K8 $\beta$ intron. By interacting with SRSF3, ORF57 affects the SRSF3-RNA interaction and attenuates the ability of SRSF3 to suppress splicing, thereby enhancing K8 $\beta$ RNA splicing (Fig. 6C). However, ORF57-mediated splicing of K8 $\beta$ RNA can be blocked by an excessive amount of RBM15 which prevents the ORF57-SRSF3 interaction and blocks ORF57 from associating with the K8 $\beta$ intron (Fig. 6C).

SRSF3 also interacts with ORF57 homologs from other members of the herpesvirus family, including herpes simplex virus type 1 (HSV-1) ICP27 (Sciabica et al. 2003), Epstein-Barr virus (EBV) SM/ EB2 (Verma et al. 2010; Juillard et al. 2012), and varicella zoster virus (VZV) IE4 protein (Ote et al. 2009). However, there are several noticeable differences in the consequences of these interactions. ICP27 binds the hypophosphorylated form of SRSF3 and SRSF7(9G8) (Escudero-Paunetto et al. 2010) to promote the export of viral transcripts. SM/EB2 counteracts the negative effect on SRSF3 on several intronless mRNAs, and the SM/EB2-SRSF3 interaction promotes nucleo-cytoplasmic export of SM/EB2-dependent, intronless viral and nonviral transcripts (Juillard et al. 2012). The SM/EB2-SRSF3 interaction also exerts a synergistic role in SM-mediated alternative splicing of STAT1 mRNA (Verma and Swaminathan 2008; Verma et al. 2010). The functional consequence of the IE4 interaction with SRSF3 was not determined (Ote et al. 2009). Together, these data all support the involvement of SRSF3 in functions of different ORF57 homologs through protein-protein interactions, although these ORF57 homologs are not interchangeable among different herpesviruses (Moriuchi et al. 1994; Han and Swaminathan 2006).

In this study, we found that ORF57 promotes RNA splicing by interacting with SRSF3 and attenuating its suppressive 
activity as exemplified in the splicing of KSHV K $8 \beta$ and BPV-1 and HPV16 late transcripts. The suppressive effect of SRSF3 on viral K8 splicing in KSHV lytic infection was also confirmed by knocking down SRSF3 expression (Fig. 4C) and by expression of ORF57 (Majerciak et al. 2008). This is in sharp contrast to the finding that SRSF3 enhances EBV SM/ EB2-mediated alternative splicing and SM recruits and coopts the function of SRSF3 in alternative splicing (Verma et al. 2010). In HSV-1 infection, viral ICP27 has a negative effect on the RNA splicing of host transcripts that shuts off host function (Hardy and Sandri-Goldin 1994; Majerciak et al. 2008; Verma and Swaminathan 2008). Although KSHV ORF57, EBV EB2, and HSV-1 ICP27 all interact with SRSF3, the observed differences among homologs in the family strongly suggest that SRSF3 has been evolutionarily adapted to the specific needs of each particular virus.

The inhibition of ORF57-mediated K8 $\beta$ splicing by transient expression of SPEN proteins was unexpected because two members of the SPEN family, RBM15 and OTT3, augment ORF57-dependent expression of KSHV intronless RNAs (Majerciak et al. 2011). Although host cofactors are required for ORF57 function, there is no report of the same host factor working in cooperation with ORF57, to both enhance the expression of viral intronless genes and inhibit RNA splicing of viral intron-containing genes. In the case of the intronless viral ORF59, RBM15 promotes ORF59 RNA accumulation in the nucleus, but interacts with ORF57 to prevent hyperpolyadenylation of the accumulated ORF59 RNA in the nucleus (Majerciak et al. 2011). In the present study, RBM15 inhibition of ORF57-mediated splicing was caused by competition between RBM15 and SRSF3 for binding to ORF57. As cells express more SRSF3 than RBM15, we saw that SRSF3 suppressed K8 $\beta$ RNA splicing more than RBM15 at the physiological level and during KSHV lytic infection. Although both SRSF3 and RBM15 are RNA-binding proteins and bind the $\mathrm{K} 8 \beta$ intron, they bind differently to the N-terminal NLS region of ORF57 which is required for ORF57-RNA interaction (Majerciak et al. 2006). SRSF3 binds ORF57 through its RRM domain (Fig. 3) and this interaction prevents its binding to RNA. However, RBM15 interacts with ORF57 via its C-terminal SPOC domain (Majerciak et al. 2011) and this interaction does not affect its N-terminal RRM domain to bind RNA, but would prevent ORF57 to interact with RNA (Fig. 6C) and SRSF3. Thus, ORF57 interacts independently with both SRSF3 and RBM15 to attenuate their suppression of RNA splicing. Therefore it is conceivable that ORF57 activities are regulated by ORF57-interacting cellular cofactors in a substrate- and dose-dependent manner.

A mechanism similar to our findings was proposed based on competitive binding, protein abundance, and structure in the interaction network of host and viral proteins (Franzosa and Xia 2011; Kiel et al. 2013). In this regard, we found that, although endogenous RBM15 interacted with ORF57 during KSHV lytic infection in B cells (data not shown), its levels were reduced as expression of SRSF3 increased over the time of viral lytic infection and the expression of ORF57 (Supplemental Fig. S3A). The decreased expression of RBM15 was most likely due to virus infection-induced host shutdown, because ORF57 alone had little effect on the expression of RBM15 in HEK293 cells (Supplemental Fig. S3B). However, we found that ORF57 alone could increase the expression of SRSF3 in HEK293 cells (Fig. 4; Supplemental Fig. S3B). Therefore, endogenous SRSF3 and viral ORF57 are two major interacting proteins that regulate K8 RNA splicing in B cells with KSHV lytic infection. Because SRSF3 is an oncogene essential for cancer cell proliferation and tumor induction and maintenance (Jia et al. 2010), the increase in expression of SRSF3 during KSHV lytic infection may also lead to global changes of host RNA splicing and may contribute to viral oncogenesis.

In conclusion, by identifying SRSF3 as an ORF57 cofactor, we have extended our knowledge that more host factors are involved in ORF57 function. The finding of that ORF57 perturbed SRSF3's negative regulation of RNA splicing of other transcripts suggests that the effects of ORF57 on SRSF3-dependent RNA splicing go well beyond KSHV transcripts. Other members of the SR protein family also bind ORF57 and regulate RNA splicing by binding to various RNA cis-elements (Majerciak et al. 2008). Thus, it would be in our great interest to know how ORF57 coordinates with other SR proteins to regulate RNA splicing or processing of other specific viral or cellular RNA transcripts in the course of KSHV lytic infection.

\section{MATERIALS AND METHODS}

\section{Cells}

HEK293 and HeLa cells were maintained in Dulbecco's modified Eagle medium (DMEM) supplemented with $10 \%$ fetal bovine serum (FBS). The origin and cultivation of HEK293 cells carrying a KSHV genome with a disrupted ORF57 gene $(B a c 36 \Delta 57)$ was described previously (Majerciak et al. 2007). The BCBL-1 B cell line carrying latent KSHV infection (Renne et al. 1996) was maintained in RPMI 1640 medium supplemented with 10\% FBS. KSHV lytic infection was induced by treatment with $1 \mathrm{mM}$ of valproic acid for BCBL- 1 cells and 3 $\mathrm{mM}$ of sodium butyrate for Bac $36 \Delta 57$ cells. All transfections were performed using Lipofectamine 2000 (Invitrogen) or LipoD293 DNA in vitro transfection reagent (SignaGen Laboratories).

\section{Plasmids}

Construction of the KSHV K8 $\beta$ cDNA expression vector pST3 and its derived vector pKY3 were previously described (Tang and Zheng 2002; Yamanegi et al. 2005). Plasmids pVM7 (ORF57-Flag), pVM24 (ORF57 $\Delta$ C-Flag, aa1-251), pVM89 (ORF57-Flag with mutated NLS2+3), pVM8 (ORF57-GFP), and pcDNA-ORF57 (untagged ORF57) were used to express various forms of ORF57 protein (Majerciak et al. 2006, 2008). Plasmids pRBM15-Flag, pRBM15-HA, and pOTT3-Flag were generously provided by Dr. Barbara Felber of NCI-Frederick and their construction was previously described (Lindtner et al. 2006; Uranishi et al. 2009). SRSF3 constructs pMA49 (Flag-SRSF3), pMA50 (SRSF3-3×Flag), pVM97 (Flag- 
SRSF3 $\Delta$ RS, aa 1-85), and pVM98 (Flag-SRSF3 $\Delta$ RRM, aa 86-164) were derived from pSRSF3-T7 (Caceres et al. 1997; Jia et al. 2010). Plasmid pML4 (GFP-K8 intron 2) was generated by inserting K8 intron 2 into the GFP coding region between a 162 and 163 in a pEGFP-N1 vector (Clontech). Plasmid pXLF1 containing a BPV-1 subgenome and pJR5 containing an HPV16 (pJR5) subgenome were described previously (Liu et al. 2003; Jia et al. 2009).

\section{Western blots}

The samples for Western blotting, if not stated otherwise, were prepared by direct lysis of transfected cells in SDS protein sample buffer supplemented with $5 \%(\mathrm{vol} / \mathrm{vol})$ 2-mercaptoethanol and analyzed with a standard protocol. The following antibodies were used in Western blot assays: anti-KSHV K8a (ProMab, Mab-2004017), custom rabbit and monoclonal anti-ORF57 (Majerciak et al. 2007), anti-Flag (Sigma, F1804 and F7425), anti-HA (Sigma, H3663 and H6908), anti- $\beta$-tubulin (Sigma, T5201), anti-SRSF3 (SRp20) (EMD Millipore, MABE116), anti-SRSF1 (ASF/SF2) (Zymed, 324500), and anti-RBM15 (Proteintech Group, 10587-1-AP).

\section{Immunoprecipitation}

Before IP, the cells were washed with PBS and lysed in IP buffer (50 mM HEPES [pH 7.5], 150 mM NaCl, 1 mM EDTA, 2.5 mM EGTA, $10 \%$ glycerol, $0.1 \%$ NP- 40 ) supplemented with a protease inhibitor cocktail (Roche). After brief sonication the lysates were cleared by centrifugation $(10,000 \mathrm{~g})$ at $4^{\circ} \mathrm{C}$ for $10 \mathrm{~min}$ and treated with RNase A/T1 (Ambion) for $10 \mathrm{~min}$ at room temperature. Prewashed, antibody-coated beads (Sigma or Millipore) were added to the cell lysate and incubated overnight at $4^{\circ} \mathrm{C}$. After extensive washes with IP buffer the immunoprecipitated complexes were eluted with IP buffer supplemented with $150 \mu \mathrm{g} / \mathrm{mL}$ of $3 \times$ Flag peptide (Sigma) for $2 \mathrm{~h}$ at $4^{\circ} \mathrm{C}$ or by $2 \times$ SDS protein sample buffer supplemented with $5 \%$ (vol/vol) 2-mercaptoethanol. Immunoprecipitated proteins were separated in SDS-PAGE and analyzed by mass spectrometry or Western blot.

\section{Mass spectrometry}

Proteins separated with SDS-PAGE were stained with GelCode Blue stain reagent (Thermo Scientific). Protein bands were digested ingel with trypsin and analyzed by Nano LC-MS/MS peptide sequencing technology by a service provider ProtTech.

\section{Immunofluorescent staining}

Immunofluorescent staining was performed as described (Majerciak et al. 2007). Briefly, the cells growing on glass coverslips were fixed with $4 \%$ paraformaldehyde, permeabilized with $0.5 \%$ Triton $\mathrm{X}$ 100 , blocked with $2 \%$ BSA, and incubated sequentially with primary and AlexaFluor-labeled secondary antibodies (Life Technologies). DNA was counterstained with Hoechst 33258 dye (Sigma). Images were collected with a Zeiss LSM510 META laser-scanning confocal microscope and analyzed with a LSM software (Zeiss). The following antibodies were used for cell staining: anti-Flag (Sigma, F1804 and F7425), anti-HA antibody (Sigma, H3663), and anti-SRSF2 (SC35) (Pharmigen, 65201a).

\section{siRNA knockdown}

To knock down the expression of endogenous proteins, HEK293 cells were transfected twice with $20 \mathrm{nM}$ of siGenome SMARTpool siRNAs (Dharmacon) targeting SRSF3 (M-030081-00), SRSF1 (M-018672-01), or RBM15 (L-010854-00). A nontargeting siRNA (D-001810-01) was used as a negative control.

\section{RT-PCR}

RNA was treated with Turbo DNA-free kit (Ambion) to remove DNA contamination prior to RT-PCR. Two-step RT-PCR was performed with MuLV reverse transcriptase in the presence of random hexamers, followed by PCR amplification using gene-specific primers and an AmpliTaq kit (Applied Biosystems). Parallel samples without RT were used as controls. Primers used in this study are listed in Supplemental Table S3.

\section{UV crosslinking and immunoprecipitation (CLIP)}

CLIP was carried out as described (Majerciak et al. 2006, 2011). At $24 \mathrm{~h}$ after cotransfection, the cells were washed with cold PBS, UV irradiated on ice, lysed in IP buffer, briefly sonicated, and cleared by centrifugation. RNA-protein complexes in the cell lysates were pulled down with anti-Flag M2 beads (Sigma). After proteinase $\mathrm{K}$ digestion the RNA in the pulldowns was extracted with a phenol: chloroform mixture, precipitated, and amplified with RT-PCR as described above.

\section{RNA oligo pulldown assays}

Customized 5'-biotinylated RNA oligonucleotides (Supplemental Table S2) were synthesized by Integrated DNA Technologies. RNA-protein pulldown assays were carried out as described (Jia et al. 2009). Briefly, RNA oligos were immobilized onto NeutrAvidin beads (Thermo Scientific) by incubation at $4^{\circ} \mathrm{C}$ for $2 \mathrm{~h}$. Unbounded oligos were removed with PBS washes. The wholecell extracts described above were diluted with PBS (1:1) and then mixed with the oligo-immobilized beads at $4^{\circ} \mathrm{C}$ overnight. After three washes with cold PBS, the proteins on the beads were eluted with $2 \times$ SDS protein sample buffer and analyzed by Western blot assays. The recombinant ORF57-Flag protein used in titration was expressed and purified from baculovirus.

\section{SUPPLEMENTAL MATERIAL}

Supplemental material is available for this article.

\section{ACKNOWLEDGMENTS}

We thank Michael Kruhlak of NCI for his help with confocal microscopy and Barbara Felber of NCI for providing the RBM15 and OTT3 constructs. This work was supported by the intramural research program of the National Cancer Institute of the National Institutes of Health.

Received March 23, 2014; accepted August 4, 2014. 


\section{REFERENCES}

Auyeung VC, Ulitsky I, McGeary SE, Bartel DP. 2013. Beyond secondary structure: Primary-sequence determinants license pri-miRNA hairpins for processing. Cell 152: 844-858.

Boyne JR, Colgan KJ, Whitehouse A. 2008. Recruitment of the complete hTREX complex is required for Kaposi's sarcoma-associated herpesvirus intronless mRNA nuclear export and virus replication. PLoS Pathog 4: e1000194.

Boyne JR, Jackson BR, Taylor A, Macnab SA, Whitehouse A. 2010. Kaposi's sarcoma-associated herpesvirus ORF57 protein interacts with PYM to enhance translation of viral intronless mRNAs. EMBO J 29: 1851-1864.

Caceres JF, Krainer AR. 1993. Functional analysis of pre-mRNA splicing factor SF2/ASF structural domains. EMBO J 12: 4715-4726.

Caceres JF, Misteli T, Screaton GR, Spector DL, Krainer AR. 1997. Role of the modular domains of SR proteins in subnuclear localization and alternative splicing specificity. J Cell Biol 138: 225-238.

Corbo C, Orru S, Gemei M, Noto RD, Mirabelli P, Imperlini E, Ruoppolo M, Vecchio LD, Salvatore F. 2012. Protein cross-talk in $\mathrm{CD} 133+$ colon cancer cells indicates activation of the Wnt pathway and upregulation of SRp20 that is potentially involved in tumorigenicity. Proteomics 12: 2045-2059.

Escudero-Paunetto L, Li L, Hernandez FP, Sandri-Goldin RM. 2010. SR proteins SRp20 and 9G8 contribute to efficient export of herpes simplex virus 1 mRNAs. Virology 401: 155-164.

Franzosa EA, Xia Y. 2011. Structural principles within the human-virus protein-protein interaction network. Proc Natl Acad Sci 108: 10538-10543.

Galiana-Arnoux D, Lejeune F, Gesnel MC, Stevenin J, Breathnach R, Del Gatto-Konczak F. 2003. The CD44 alternative v9 exon contains a splicing enhancer responsive to the SR proteins 9G8, ASF/SF2, and SRp20. J Biol Chem 278: 32943-32953.

Guito J, Lukac DM. 2012. KSHV Rta promoter specification and viral reactivation. Front Microbiol 3: 30.

Gupta AK, Ruvolo V, Patterson C, Swaminathan S. 2000. The human herpesvirus 8 homolog of Epstein-Barr virus SM protein (KS-SM) is a posttranscriptional activator of gene expression. J Virol 74: $1038-1044$.

Han Z, Swaminathan S. 2006. Kaposi's sarcoma-associated herpesvirus lytic gene ORF57 is essential for infectious virion production. J Virol 80: 5251-5260.

Hardy WR, Sandri-Goldin RM. 1994. Herpes simplex virus inhibits host cell splicing, and regulatory protein ICP27 is required for this effect. J Virol 68: 7790-7799.

He X, Arslan AD, Pool MD, Ho TT, Darcy KM, Coon JS, Beck WT. 2011. Knockdown of splicing factor SRp20 causes apoptosis in ovarian cancer cells and its expression is associated with malignancy of epithelial ovarian cancer. Oncogene 30: 356-365.

Hiriart E, Gruffat H, Buisson M, Mikaelian I, Keppler S, Meresse P, Mercher T, Bernard OA, Sergeant A, Manet E. 2005. Interaction of the Epstein-Barr virus mRNA export factor EB2 with human Spen proteins SHARP, OTT1, and a novel member of the family, OTT3, links Spen proteins with splicing regulation and mRNA export. $J$ Biol Chem 280: 36935-36945.

Huang Y, Steitz JA. 2001. Splicing factors SRp20 and 9G8 promote the nucleocytoplasmic export of mRNA. Mol Cell 7: 899-905.

Iborra S, Hirschfeld M, Jaeger M, Zur HA, Braicu I, Sehouli J, Gitsch G, Stickeler E. 2013. Alterations in expression pattern of splicing factors in epithelial ovarian cancer and its clinical impact. Int J Gynecol Cancer 23: 990-996.

Jia R, Zheng ZM. 2012. SRSF3 (serine/arginine-rich splicing factor 3). Atlas Genet Cytogenet Oncol Haematol. http://AtlasGenetics Oncology.org/Genes/SRSF3ID42279ch6p21.html.

Jia R, Liu X, Tao M, Kruhlak M, Guo M, Meyers C, Baker CC, Zheng ZM. 2009. Control of the papillomavirus early-to-late switch by differentially expressed SRp20. J Virol 83: 167-180.

Jia R, Li C, McCoy JP, Deng CX, Zheng ZM. 2010. SRp20 is a proto-oncogene critical for cell proliferation and tumor induction and maintenance. Int J Biol Sci 6: 806-826.
Juillard F, Bazot Q, Mure F, Tafforeau L, Macri C, Rabourdin-Combe C, Lotteau V, Manet E, Gruffat H. 2012. Epstein-Barr virus protein EB2 stimulates cytoplasmic mRNA accumulation by counteracting the deleterious effects of SRp20 on viral mRNAs. Nucleic Acids Res 40: 6834-6849.

Jumaa H, Nielsen PJ. 1997. The splicing factor SRp20 modifies splicing of its own mRNA and ASF/SF2 antagonizes this regulation. EMBO J 16: 5077-5085.

Jumaa H, Wei G, Nielsen PJ. 1999. Blastocyst formation is blocked in mouse embryos lacking the splicing factor SRp20. Curr Biol 9: 899-902.

Kang JG, Pripuzova N, Majerciak V, Kruhlak M, Le SY, Zheng ZM. 2011. Kaposi's sarcoma-associated herpesvirus ORF57 promotes escape of viral and human interleukin-6 from microRNA-mediated suppression. J Virol 85: 2620-2630.

Kiel C, Verschueren E, Yang JS, Serrano L. 2013. Integration of protein abundance and structure data reveals competition in the ErbB signaling network. Sci Signal 6: ra109.

Kirshner JR, Lukac DM, Chang J, Ganem D. 2000. Kaposi's sarcomaassociated herpesvirus open reading frame 57 encodes a posttranscriptional regulator with multiple distinct activities. $J$ Virol 74: 3586-3597.

Lindtner S, Zolotukhin AS, Uranishi H, Bear J, Kulkarni V, Smulevitch S, Samiotaki M, Panayotou G, Felber BK, Pavlakis GN. 2006. RNA-binding motif protein 15 binds to the RNA transport element RTE and provides a direct link to the NXF1 export pathway. $J$ Biol Chem 281: 36915-36928.

Liu X, Mayeda A, Tao M, Zheng ZM. 2003. Exonic splicing enhancerdependent selection of the bovine papillomavirus type 1 nucleotide $32253^{\prime}$ splice site can be rescued in a cell lacking splicing factor ASF/ SF2 through activation of the phosphatidylinositol 3-kinase/Akt pathway. J Virol 77: 2105-2115.

Loomis RJ, Naoe Y, Parker JB, Savic V, Bozovsky MR, Macfarlan T, Manley JL, Chakravarti D. 2009. Chromatin binding of SRp20 and ASF/SF2 and dissociation from mitotic chromosomes is modulated by histone H3 serine 10 phosphorylation. Mol Cell 33: 450-461.

Lou H, Neugebauer KM, Gagel RF, Berget SM. 1998. Regulation of alternative polyadenylation by U1 snRNPs and SRp20. Mol Cell Biol 18: $4977-4985$.

Maciolek NL, McNally MT. 2007. Serine/arginine-rich proteins contribute to negative regulator of splicing element-stimulated polyadenylation in Rous sarcoma virus. J Virol 81: 11208-11217.

Majerciak V, Zheng ZM. 2009. Kaposi's sarcoma-associated herpesvirus ORF57 in viral RNA processing. Front Biosci 14: 1516-1528.

Majerciak V, Yamanegi K, Nie SH, Zheng ZM. 2006. Structural and functional analyses of Kaposi sarcoma-associated herpesvirus ORF57 nuclear localization signals in living cells. J Biol Chem 281: 28365-28378.

Majerciak V, Pripuzova N, McCoy JP, Gao SJ, Zheng ZM. 2007. Targeted disruption of Kaposi's sarcoma-associated herpesvirus ORF57 in the viral genome is detrimental for the expression of ORF59, K8a, and K8.1 and the production of infectious virus. $J$ Virol 81: 1062-1071.

Majerciak V, Yamanegi K, Allemand E, Kruhlak M, Krainer AR, Zheng ZM. 2008. Kaposi's sarcoma-associated herpesvirus ORF57 functions as a viral splicing factor and promotes expression of intron-containing viral lytic genes in spliceosome-mediated RNA splicing. J Virol 82: 2792-2801.

Majerciak V, Uranishi H, Kruhlak M, Pilkington GR, Massimelli MJ, Bear J, Pavlakis GN, Felber BK, Zheng ZM. 2011. Kaposi's sarcoma-associated herpesvirus ORF57 interacts with cellular RNA export cofactors RBM15 and OTT3 to promote expression of viral ORF59. J Virol 85: 1528-1540.

Malik P, Clements JB. 2004. Protein kinase CK2 phosphorylation regulates the interaction of Kaposi's sarcoma-associated herpesvirus regulatory protein ORF57 with its multifunctional partner hnRNP K. Nucleic Acids Res 32: 5553-5569.

Malik P, Blackbourn DJ, Clements JB. 2004. The evolutionarily conserved Kaposi's sarcoma-associated herpesvirus ORF57 protein 
interacts with REF protein and acts as an RNA export factor. $J$ Biol Chem 279: 33001-33011.

Manley JL, Krainer AR. 2010. A rational nomenclature for serine/arginine-rich protein splicing factors (SR proteins). Genes Dev 24: 1073-1074.

Massimelli MJ, Kang JG, Majerciak V, Le SY, Liewehr DJ, Steinberg SM, Zheng ZM. 2011. Stability of a long noncoding viral RNA depends on a 9-nt core element at the RNA $5^{\prime}$ end to interact with viral ORF57 and cellular PABPC1. Int J Biol Sci 7: 1145-1160.

Massimelli MJ, Majerciak V, Kruhlak M, Zheng ZM. 2013. Interplay between polyadenylate-binding protein 1 and Kaposi's sarcoma-associated herpesvirus ORF57 in accumulation of polyadenylated nuclear RNA, a viral long noncoding RNA. J Virol 87: 243-256.

Moriuchi H, Moriuchi M, Smith HA, Cohen JI. 1994. Varicella-zoster virus open reading frame 4 protein is functionally distinct from and does not complement its herpes simplex virus type 1 homolog, ICP27. J Virol 68: 1987-1992.

Nekorchuk M, Han Z, Hsieh TT, Swaminathan S. 2007. Kaposi's sarcoma-associated herpesvirus ORF57 protein enhances mRNA accumulation independently of effects on nuclear RNA export. J Virol 81: 9990-9998.

Ote I, Lebrun M, Vandevenne P, Bontems S, Medina-Palazon C, Manet E, Piette J, Sadzot-Delvaux C. 2009. Varicella-zoster virus IE4 protein interacts with SR proteins and exports mRNAs through the TAP/NXF1 pathway. PLoS One 4: e7882.

Pilkington GR, Majerciak V, Bear J, Uranishi H, Zheng ZM, Felber BK. 2012. Kaposi's sarcoma-associated herpesvirus ORF57 is not a bona fide export factor. J Virol 86: 13089-13094.

Renne R, Zhong W, Herndier B, McGrath M, Abbey B, Kedes D, Ganem D. 1996. Lytic growth of Kaposi's sarcoma-associated herpesvirus (human herpesvirus 8) in culture. Nat Med 2: 342-346.

Saeki K, Yasugi E, Okuma E, Breit SN, Nakamura M, Toda T, Kaburagi Y, Yuo A. 2005. Proteomic analysis on insulin signaling in human hematopoietic cells: identification of CLIC1 and SRp20 as novel downstream effectors of insulin. Am J Physiol Endocrinol Metab 289: E419-E428.
Sciabica KS, Dai QJ, Sandri-Goldin RM. 2003. ICP27 interacts with SRPK1 to mediate HSV splicing inhibition by altering SR protein phosphorylation. EMBO J 22: 1608-1619.

Sen S, Talukdar I, Webster NJ. 2009. SRp20 and CUG-BP1 modulate insulin receptor exon 11 alternative splicing. Mol Cell Biol 29: 871-880.

Sen S, Jumaa H, Webster NJ. 2013. Splicing factor SRSF3 is crucial for hepatocyte differentiation and metabolic function. Nat Commun 4: 1336.

Tang S, Zheng ZM. 2002. Kaposi's sarcoma-associated herpesvirus K8 exon 3 contains three $5^{\prime}$-splice sites and harbors a K8.1 transcription start site. J Biol Chem 277: 14547-14556.

Uranishi H, Zolotukhin AS, Lindtner S, Warming S, Zhang GM, Bear J, Copeland NG, Jenkins NA, Pavlakis GN, Felber BK. 2009. The RNA binding motif protein 15B (RBM15B/OTT3) acts as cofactor of the nuclear export receptor NXF1. J Biol Chem 284: 26106-26116.

Verma D, Swaminathan S. 2008. Epstein-Barr virus SM protein functions as an alternative splicing factor. J Virol 82: 7180-7188.

Verma D, Bais S, Gaillard M, Swaminathan S. 2010. Epstein-Barr virus SM protein utilizes cellular splicing factor SRp20 to mediate alternative splicing. J Virol 84: 11781-11789.

Watanuki T, Funato H, Uchida S, Matsubara T, Kobayashi A, Wakabayashi Y, Otsuki K, Nishida A, Watanabe Y. 2008. Increased expression of splicing factor SRp20 mRNA in bipolar disorder patients. J Affect Disord 110: 62-69.

Wong J, Garner B, Halliday GM, Kwok JB. 2012. Srp20 regulates TrkB pre-mRNA splicing to generate TrkB-Shc transcripts with implications for Alzheimer's disease. J Neurochem 123: 159-171.

Yamanegi K, Tang S, Zheng ZM. 2005. Kaposi's sarcoma-associated herpesvirus $\mathrm{K} 8 \beta$ is derived from a spliced intermediate of K8 pre-mRNA and antagonizes K8a (K-bZIP) to induce p21 and p53 and blocks K8a-CDK2 interaction. J Virol 79: 14207-14221.

Zahler AM, Lane WS, Stolk JA, Roth MB. 1992. SR proteins: a conserved family of pre-mRNA splicing factors. Genes Dev 6: 837-847.

Zhou Z, Licklider LJ, Gygi SP, Reed R. 2002. Comprehensive proteomic analysis of the human spliceosome. Nature 419: 182-185. 

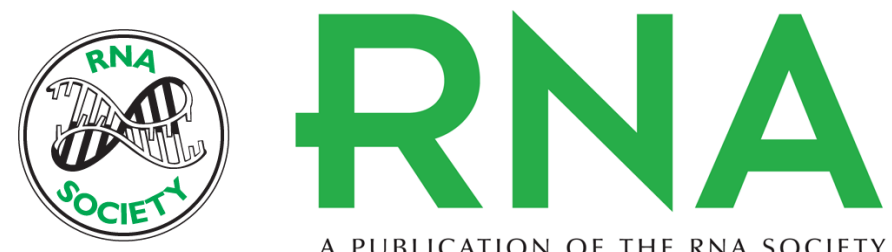

A PUBLICATION OF THE RNA SOCIETY

\section{Attenuation of the suppressive activity of cellular splicing factor SRSF3 by Kaposi sarcoma-associated herpesvirus ORF57 protein is required for RNA splicing}

Vladimir Majerciak, Mathew Lu, Xiaofan Li, et al.

RNA 2014 20: 1747-1758 originally published online September 18, 2014

Access the most recent version at doi:10.1261/rna.045500.114

Supplemental Material

References

Open Access

Creative Commons License

Email Alerting Service
http://rnajournal.cshlp.org/content/suppl/2014/09/03/rna.045500.114.DC1

This article cites 58 articles, 36 of which can be accessed free at: http://rnajournal.cshlp.org/content/20/11/1747.full.html\#ref-list-1

Freely available online through the RNA Open Access option.

This article, published in RNA, is available under a Creative Commons License (Attribution-NonCommercial 4.0 International), as described at http://creativecommons.org/licenses/by-nc/4.0/.

Receive free email alerts when new articles cite this article - sign up in the box at the top right corner of the article or click here.

\section{|||||||| Providing Precise Solutions for} your research.

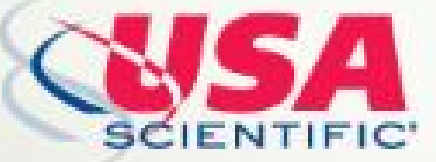

To subscribe to $R N A$ go to:

http://rnajournal.cshlp.org/subscriptions 